\title{
LAS POLÍTICAS CULTURALES Y EL CONCEPTO DE CULTURA. Etnografía de un evento festivo intercultural
}

\author{
Antonia OLMOS ALCARAZ; María RUBIO GÓMEZ; Pierangela CONTINI \\ Universidad de Granada (España) \\ antonia@ugr.es, marrubio82@gmail.com, pierangela.contini@gmail.com
}

\begin{abstract}
CULTURAL AND POLITICAL CONCEPT OF CULTURE. Ethnography of an intercultural festive event
\end{abstract}

Resumen: En el contexto de estudio de este trabajo, la diversidad cultural ha sido gestionada a través de políticas públicas en materia de educación, salud, trabajo y servicios sociales, desde modelos integracionistas. A nivel local la atención se ha centrado más en la implementación de "políticas culturales", cuyo objetivo es promover la convivencia cotidiana en escenarios de diversidad. El presente artículo ofrece una reflexión sobre las políticas culturales desarrolladas a nivel local relacionadas con la convivialidad y la diversidad, a través del análisis de un ejemplo concreto de práctica tipificada como intercultural en la ciudad de Granada.

Para ello, ofrecemos una descripción detallada de sus distintos momentos reflexionando sobre el concepto de interculturalidad y de cultura subyacentes y analizamos, con la ayuda del material etnográfico, qué efectos o calado pueden tener esta tipología de efeméride en la población local y de qué manera ésta se ve representada. Como conclusión se reportan algunas propuestas nacidas de los/as propios/as implicados/as, sobre cómo seguir implementando políticas culturales que puedan ofrecer respuestas a las demandas sociales y representar a toda la comunidad.

Abstract: The public policies on education, health, work, social services, etc, have been used by many States in order to manage cultural diversity by using integrationist models. At a local level the attention has been focused more on the implementation of "cultural policies" aimed at promoting the daily coexistence in diversity scenarios.

This article offers a reflection on cultural policies developed locally related to conviviality and diversity, through the analysis of "The Festival of Cultures", a particular example of a practice defined as intercultural in the city of Granada.

In order for this to be accomplished, we offer a detailed description of its different moments reflecting on the concept of multiculturalism and underlying culture and analyzing, with the help of ethnographic material, which effects or consequences can this type of festivals have in the local population and how this is represented.

Concluding, some proposals arose from the people involved are reported, on how to keep implementing cultural policies that can provide answers to social demands and represent the entire community.

Palabras clave: Políticas culturales. Interculturalidad. Diversidad cultural. Celebración intercultural. Granada Cultural policies. Interculturality. Cultural diversity. Intercultural Celebrations. 


\section{Introducción ${ }^{1}$}

La creciente diversidad que caracteriza a muchas ciudades europeas en este momento, fruto en parte de las actuales migraciones internacionales, ha sido abordada por las Ciencias Sociales principalmente en términos de integración, asimilación y adaptación de la población inmigrante a las "sociedades mayoritarias" (Oliveira y Padilla, 2012; Castells y Miller, 2003). Es por ello que no se ha puesto demasiado énfasis en el análisis de las dinámicas de convivencia cotidiana generadas a nivel local en barrios y ciudades en el marco de una nueva "diversidad de diversidades" (Vertovec, 2007; Meissner y Vertovec, 2015; Padilla, Azevedo y Olmos Alcaraz, 2015)².

En este escenario las políticas públicas sobre educación, salud, trabajo, servicios sociales, etc. diseñadas para funcionar como "herramientas de integración", vienen siendo promovidas desde los Estados con el objetivo de gestionar la diversidad cultural; sin embargo, es desde el nivel local (Ayuntamientos, Diputaciones, asociaciones e instituciones de ámbito municipal) que, a través de la implementación de lo que se conoce como "políticas culturales", se inician planes y programas para fomentar la convivencia cotidiana en escenarios de diversidad (Clark y Navarro, 2009; Oliveira y Padilla, 2012; Ray, 2003). Muchos de estos planes y programas son, a menudo, definidos como interculturales.

Pero no siempre está claro a qué nos referimos cuando hablamos de políticas culturales ${ }^{3}$. Por establecer unos mínimos a partir de los que iniciar nuestros análisis, podemos definir las mismas como un conjunto estructurado de acciones y prácticas sociales generadas y desarrolladas por organismos públicos y/o privados y otros agentes sociales en el ámbito de la cultura. Como sugieren Nicolás Barbieri, Adriana Partal y Eva Merino:

"Analizar el campo de las políticas públicas culturales implica enfrentarse, en primer lugar, a un reto conceptual. Delimitar, aunque solo sea de forma operacional, el campo de la cultura y el de las políticas públicas que se pretende estudiar es enfrentarse con palabras que nacen y evolucionan en situaciones cambiantes relacionadas con el ejercicio del poder. Cada aplicación del término cultura (y del de política cultural) implica su redefinición, y el desgaste facilita su utilización poco rigurosa y a veces incluso demagógica" (Barbieri et al., 2011: 478).

La indefinición de los conceptos a la hora de referirse a la política cultural hace que ésta se presente como un puente "entre los registros estético y antropológico" (Miller y Yúdice, 2004 citado en Barbieri et al., 2011: 478). Y en este puente el concepto de cultura es el más

\footnotetext{
1 El presente texto forma parte de algunas de las conclusiones del proyecto de investigación "Culturas de Convivência e Super-diversidade" (PTDC/CS-SOC/101693/2008), financiado por la Fundação para a Ciência e a Tecnologia de Portugal y dirigido por la profesora Beatriz Padilla con la participación del instituto de Migraciones de la Universidad de Granada. Agradecemos a la citada fundación del apoyo económico para el desarrollo del trabajo de campo puesto en marcha en la mencionada investigación. Igualmente forma parte del la investigación para la obtención del grado de doctorado de Pierangela Contini, desarrollada en el Departamento de Antropología Social y en el Instituto de Migraciones de la Universidad de Granada.

2 En otros trabajos hemos reflexionado sobre estas dinámicas generadas "desde abajo" en el ámbito local (Contini, 2015), preocupándonos de observar cómo la manera de entender los conceptos de diversidad, cultura, interculturalidad (y otros términos asociados), tiene influencias concretas tanto en los discursos como en las practicas de convivencia cotidiana (García Castaño, Olmos Alcaraz, Contini y Rubio Gómez, 2011; García Castaño, Olmos Alcaraz y Contini, 2012; Olmos y Contini, en prensa; Contini y Olmos, 2015).

3 Para profundizar sobre los debates conceptuales actuales existentes en torno las políticas culturales pueden consultarse los trabajos del Real Instituto Elcano de Estudios Internacionales y Estratégicos (2004), Rodríguez Morató (2012) y Zallo (2011).
} 
flexible, ambiguo y menos definido. La cuestión finalmente reside en cómo es entendida ésta última: en su versión más restringida, estática y cosificada (como folklore, arte, artesanía, gastronomía y demás elementos objetivados); o como proceso en continuo movimiento, en tanto que universos simbólicos compartidos, creados y recreados continuamente por una comunidad. Si nos situamos en esta última definición, hablar de cultura sería hablar de diversidad cultural.

Por otro lado, hemos de reparar en que las políticas culturales se fijan unos objetivos explícitos y otros que no lo son tanto. En este sentido Zallo nos habla de "objetivos implícitos como son los de identidad nacional -ámbito de distinta percepción en las nacionalidades históricas- y la diversidad cultural e institucional" (Zallo, 2011: 57). No obstante, es a la diversidad cultural a la que las instituciones dedican tantos esfuerzos, siendo el objetivo explícito principal su gestión y (re)organización. En este ámbito, tanto desde las administraciones públicas como desde el tercer sector, los discursos y las prácticas interculturales casi siempre se encuentran relacionados con temas "migratorios" (García et al., 2011). Generalmente lo intercultural se suele abordar junto a cuestiones relativas a la inserción-inclusión-integración social y laboral de la población inmigrante dentro de la sociedad definida como mayoritaria. Para lograr los objetivos mencionados, las administraciones públicas y demás asociaciones e instituciones implicadas en la gestión de la diversidad recurren a varias medidas relacionadas con la sensibilización, la promoción del diálogo, la mediación o la educación, que son apellidadas de "interculturales". Es así como nos encontramos con la mediación intercultural, educación intercultural, etc., todo ello principalmente implementado en el ámbito de lo local (ibídem).

Con estas premisas resulta imperativo evidenciar el rol que juegan las políticas culturales (especialmente) de ámbito local en la promoción e implementación de acciones de cuño cultural, prestando especial atención a la manera en que éstas entienden la diversidad: ¿qué diversidad y qué cultura es la que pretenden promocionar? Y en caso de utilizar un enfoque intercultural: ¿a que interculturalismo se están refiriendo?

El presente artículo ofrece una reflexión sobre las políticas culturales desarrolladas a nivel local, relacionadas con la convivialidad y la diversidad, a través del análisis de un ejemplo concreto de práctica tipificada como intercultural en un contexto determinado: la ciudad de Granada. Para ello se han observado las distintas fases (concepción, planificación y realización) de un evento conocido como Fiesta de las Culturas ${ }^{4}$, que lleva celebrándose en la ciudad desde hace algo más de 15 años. Trataremos de realizar una descripción detallada de sus distintos momentos para, entre otras cosas, extraer el concepto de interculturalidad y de cultura subyacentes y reflexionar, con la ayuda del material etnográfico, sobre qué efectos o calado tiene en la población local y cómo ésta la experimenta o se ve representada en dichas acciones. Por último, también analizaremos una serie de propuestas, nacidas de las personas implicadas, sobre cómo seguir implementando políticas culturales que den respuesta a las demandas sociales y que representen a toda la comunidad.

Pero antes de comenzar con la descripción del evento consideramos importante posicionarnos sobre algo que nos parece fundamental en la materia: la población a la que van dirigidas estas acciones. Huelga decir que hoy por hoy Granada, España, Europa, son sociedades multiculturales -si es que acaso alguna vez no lo fueron-, pero es importante señalar que no todas las diversidades que integran esa multiculturalidad son igualmente visibilizadas, valoradas y reconocidas socialmente. Granada se nutre de muchos tipos de población:

4 Para mantener el anonimato de quienes participaron en nuestra investigación hemos sustituidos por nombres ficticios los nombres propios que aparecen en el texto (de personas, lugares, asociaciones y del mismo evento). Pero es útil especificar que en el nombre real del evento de casi todas las ediciones se ha hecho referencia a la interculturalidad y a la convivencia, de lo que se intuyen las intenciones y los objetivos que la entidad organizadora se prefije con la realización de la fiesta. 
turistas de todo el mundo, estudiantes nacionales e internacionales, población trabajadora inmigrante extranjera europea y extra-comunitaria; pero también cuenta con una importante comunidad gitana con presencia histórica en el territorio; con poblaciones de todos los estratos sociales, familias enteras, personas mayores y jóvenes; musulmanes, cristianos, evangélicos y budistas, entre otras confesiones religiosas. Podríamos no terminar nunca si el objetivo es enumerar todos los tipos de diversidades y si por diversidades entendemos, como nos insiste Santos (2011), la diversidad infinita y característica del mundo.

Granada es, por lo tanto, una ciudad súper-diversa, en los términos que nos explica Vertovec (2007), dado que la diversidad no puede limitarse a lo étnico. Y decíamos posicionarnos porque, a pesar de esta heterogeneidad de población, las políticas culturales en la ciudad son bastante homogéneas. La inmensa mayoría van dirigidas a unos determinados sectores de la población autóctona, como bien nos señalaba el siguiente informante:

... vamos a decirlo claro, el Ayuntamiento tendrá potestad para decidir en qué va a invertir su dinero: ¡en la gente de Granada! y de hecho para ser más claro todavía, el eslogan político de la campaña electoral del Partido Popular, que es el partido que ahora mismo está al poder en el Ayuntamiento de Granada, es: "primero, Granada” ¡más claro que el sol, agua! (Organizador del evento, Granada 2011).

Y de manera muy residual encontramos lo que hoy estamos analizando aquí, acciones y eventos donde tienen presencia otras poblaciones. Para nosotros esto no se corresponde con una verdadera política cultural, cuyo objetivo debiera ser gestionar conjuntamente una diversidad que nadie ha vivido antes (Zapata-Barrero, 2010), ni las poblaciones autóctonas ni los recién -o no tanto- llegados.

\section{Descripción metodológica}

Para la elaboración de este artículo hemos utilizado los datos generados en el marco del proyecto de investigación "Culturas de Convivência e Super- diversidade" (PTDC/CSSOC/101693/2008), financiado por la Fundação para a Ciência e a Tecnologia de Portugal, y desarrollado conjuntamente entre el Instituto de Migraciones de la Universidad de Granada y el CIES-ISCTE de Lisboa, entre los años 2009 y 2012.

Con el proyecto se aspiraba a abordar las dinámicas cotidianas convivenciales de dos ciudades europeas significadas por una importante presencia de "nuevos vecinos" de diversa procedencia geográfica/nacional: Granada y Lisboa, observando en detalle en cada una de las ciudades un barrio céntrico y otro periférico. Para ello, el proyecto se centró en el estudio de distintos contextos de las ciudades, tanto informales como formales (Lofland, 1998), realizando trabajo de campo etnográfico principalmente a través de observación participante y entrevistas abiertas. Cuando nos referimos a "contextos informales" aludimos a la observación realizada en los barrios, en concreto sobre los procesos cotidianos allí acontecidos y las dinámicas establecidas entre los distintos actores sociales (residentes, comerciantes, representantes institucionales, entre otros) presentes en los mismos: en calles, plazas, parques, mercados, etc. Los contextos definidos como "formales" por el proyecto fueron principalmente escuelas y edificios/sedes de las distintas dependencias del gobierno municipal; pero también eventos organizados y pautados por los distintos agentes implicados en la gestión pública local, como el evento intercultural que analizamos en este artículo. Es decir, nuestra división entre "contexto informal" y "contexto formal" no remite exclusivamente a dimensiones geo-espaciales, sino que implica también cierta organización y previsión en el desarrollo de las interacción sociales a las que remite sin circunscribirnos a un espacio físico concreto. 
El trabajo de campo en estos últimos contextos se llevó a cabo más tarde, tras un periodo de tiempo en el campo observando los espacios definidos como "informales". Siendo así, en el caso de la ciudad de Granada -que es el que analizamos en este artículo- realizamos diversas aproximaciones a escuelas e institutos de educación secundaria, hasta finalmente realizar observación participante y entrevistas de manera más intensa en un solo un centro, en este caso de educación secundaria. Para la selección del evento intercultural (como expresión de las políticas culturales puestas en marcha a nivel local para la promoción de la diversidad cultural), se elaboró un registro con los datos de los eventos interculturales más relevantes del área metropolitana de Granada, listando de cada evento datos como la fecha y área de realización, ubicación en la ciudad, entidad organizadora (asociaciones de vecinos, Ayuntamiento, asociaciones y ONG) y beneficiarios. El evento elegido para la investigación fue la Fiesta de las Culturas, organizado por la Plataforma pro-inmigrantes ${ }^{5}$ en la que participan varias asociaciones y entidades vinculadas con la inmigración en Granada. Para el estudio de esta efeméride se procedió participado en las reuniones organizativas, en la que se realizaron un total de 14 observaciones protocolizadas y 11 entrevistas formales abiertas. 5 de estas entrevistas se realizaron a organizadores del evento y 6 a participantes a lo largo del día de la celebración. Además, contamos con conversaciones no formales realizadas tanto a lo largo del trabajo de campo, como el día del evento, a participantes y vecinos del área donde la actividad tuvo lugar. Durante el trabajo de campo se produjeron documentos relacionados con la organización y realización del evento (periódicos, documentos de trabajo internos, datos e informes de anteriores celebraciones, información estadística, etc.) utilizando para el análisis interpretativo de todos los datos producidos el programa informático NVivo.

El evento analizado, la Fiesta de las Culturas, no cuenta con un emplazamiento estable para su celebración anual, sino que se ha ido realizando en lugares distintos de la ciudad en cada edición. En el periodo de desarrollo de nuestro proyecto de investigación, el espacio elegido para la celebración de la misma fue la zona centro de la ciudad. Pero vayamos a conocer un poco más cómo surge, se gesta y se va haciendo visible a través de los años esta política intercultural en la ciudad de Granada.

\section{La fiesta de las culturas: análisis de una política cultural implementada a nivel local}

La primera Fiesta de las Culturas se celebró en el año 1995 por iniciativa de la recién creada por entonces Plataforma pro-inmigrantes ${ }^{6}$. Se trata del evento intercultural con más repercusión en la ciudad de Granada con sus 15 ediciones con cadencia anual y la participación de diversas asociaciones y entidades vinculadas con la inmigración en la ciudad, así como una importante cantidad de vecinos y curiosos ocasionales para asistir a espectáculos varios (bailes, performances, talleres, cata de gastronomía, etc.) de corte folclórico fundamentalmente, pero con un claro componente reivindicativo de los derechos de las personas inmigrantes. De hecho fue la primera actividad que la Plataforma organizó y que ha tenido continuidad en el tiempo:

[...] la Fiesta de las Culturas es la primera actividad que salió y se mantuvo desde la Plataforma pro-inmigrantes sigue estando allí, como es una actividad base, que refleja mucho la esencia de la Plataforma, y es una fiesta en la que

5 La Plataforma pro-inmigrantes es una agrupación que desde 1993 reúne ONG, asociaciones y entidades públicas y privadas con el objetivo de trabajar en materia de reconocimiento de los derechos de los inmigrantes desde el plano municipal.

6 La Plataforma pro-inmigrantes es una agrupación que desde 1993 reúne ONG, asociaciones y entidades públicas y privadas con el objetivo de trabajar en materia de reconocimiento de los derechos de los inmigrantes desde el plano municipal. 
participan varias asociaciones de inmigrantes y pro-inmigrantes, como es el caso de ASPA, para darse a conocer, para visibilizar el hecho de la convivencia con personas de otras culturas, de manera positiva (Organizador del evento, Granada 2011).

Esta casuística nos da ya algunas pistas sobre la concepción del evento desde sus orígenes y sobre las prioridades de la entidad organizadora con respecto a las temáticas de inmigración. Se trata de una iniciativa sustentada principalmente por entidades del tercer sector cuya filosofía desde un principio fue, como nos narraba uno de los organizadores, eminentemente reivindicativa con respecto a los derechos de las personas inmigrantes:

[...] siempre ha tenido un carácter muy reivindicativo, las primeras campañas de derecho al voto con los inmigrantes, hace ya 12 años también estuvieron alli presentes [en la fiesta]. Siempre ha tenido cartel y contenido, siempre ha sido una preocupación básica nuestra: hay que darle contenido, y también ha habido discusión entre multiculturalidad y ciudadanía, todo esto[...] (Organizador del evento, Granada 2011b).

Es decir, el foco de atención se pone en la población inmigrante, por lo tanto se fija solo en una parte de esa diversidad de la que hablábamos. Iremos retomando esta idea a lo largo de todo el artículo. Pero nos interesaba también saber qué papel jugaba y juega la administración pública local tanto en la organización misma del evento, como en el apoyo institucional al mismo. En este sentido nuestras observaciones y las entrevistas realizadas han constatado que ese apoyo ha sido puntual a lo largo de la historia de la fiesta:

El ayuntamiento al principio [PSOE] daba un poco de apoyo económico, nunca ha sido una idea que los ha ilusionado o animado demasiado pero había una aceptación, pero en los momentos más difíciles [...] hemos tenido problemas con el PP (Organizador del evento, Granada 2011b).

Actualmente dicho apoyo es inexistente, dado que depende mucho de qué partido esté gobernando en ese momento. Como nos decía uno de los organizadores "la falta de relación con la administración local ha sido el principal problema y dificultad" (Organizador del evento, Granada 2011b).

\subsection{Planificación y desarrollo del evento}

Una vez esbozados los planteamientos básicos que sustentan el evento, pasamos a describir cómo ha acontecido la planificación y desarrollo del mismo en la edición observada. Y lo primero que queremos destacar es la labor de quienes diseñan, planifican e implementan la fiesta. En la negociación previa a la planificación del evento llevan la voz cantante las ONG de "defensa del inmigrante", no "asociaciones de inmigrantes". Éstas últimas son invitadas a participar pero, como hemos dicho anteriormente, con unas normas pre-establecidas por las ONG pro-inmigrantes. Esta situación no es del gusto de algunas asociaciones de inmigrantes, que manifiestan abiertamente su interés porque el evento sea gestionado por ellos mismos:

[...] ha habido un momento de la Plataforma en el que había 40 o más asociaciones y la mitad de ellas eran instituidas por migrantes. Pero hubo conflictos porque algunos de ellos querían 'apartar' a las demás asociaciones constituidas por no inmigrantes (Observación, Granada 2011). 
La Plataforma escucha sugerencias en este y otros sentidos, pero la gestión del evento sigue siendo responsabilidad de las asociaciones y ONG con mayor protagonismo y de quienes llevan más tiempo formando parte de la misma, y éstas no son las formadas por inmigrantes, aunque en algún momento hayan sido muy numerosas.

En cuanto a la estructura que sigue la Fiesta de las Culturas año tras año, hay que decir que, a día de hoy, es más o menos estable con respecto a las actividades que la integran y la forma de organizarlas, fruto de la experiencia de ediciones anteriores. Esto es visto por organizadores y participantes como un punto fuerte de la misma, dado que se considera que si se hiciese de otra manera se generaría en la población una sensación de incertidumbre propia de "las cosas puntuales y distintas, sin continuidad en el tiempo" (Organizador del evento, Granada 2011).

Normalmente el evento consta, en primer lugar, de una serie de stands informativos de las ONG, asociaciones y demás entidades que participan; y stands con información de países, regiones o colectivos en concreto -por ejemplo, en la edición estudiada había también un stand del colectivo gitano, dedicado a confeccionar con distintos materiales una bandera gitana-.

Por otro lado, se organizan talleres de diversa índole, donde los asistentes a la fiesta están invitados a participar. Diseñados e implementados por los diversos colectivos que toman parte en la fiesta, dichos talleres están orientados a niños, jóvenes y personas mayores, es decir a todos los vecinos y vecinas que quieran acercarse. Incluso se pretende que se involucren personas de distintas edades conjuntamente en los mismos. Las temáticas que se trabajan en esta actividad son de lo más diverso: desde talleres de realización de henna ${ }^{7}$, donde mujeres marroquíes dibujan las pieles de aquellas personas que lo desean, pasando por talleres de papiroflexia o de elaboración de flores de papel, hasta talleres de maquillaje, juegos del mundo y manualidades varias. La idea es que los asistentes pasen toda la jornada interaccionando entre sí con la excusa de su participación en los talleres.

Otra de las actividades habituales de la Fiesta de las Culturas es la toma del conocido y tradicional "té intercultural". Desde los inicios de la fiesta, pasadas las primeras horas del evento, cuando los asistentes ya han visto y reconocido los stands, se viene ofreciendo té y pastas marroquíes -aunque la organización de la fiesta lo denomina el "té intercultural" dado que pretenden con ello que todas las personas participantes se relacionen-.

El folklore en general tiene un papel central en todo el evento. Los bailes tradicionales son también un habitual de la Fiesta. De hecho suelen ser el "plato fuerte", en el sentido de que son una de las actividades que más público tienen y que, según asistentes y organizadores, más gustan. Pero hay un poco de todo: música y baile, trajes y banderas, juegos y artesanía... y todo ello de corte "nacional". Es decir, los colectivos se organizan principalmente según sus procedencias ${ }^{8}$, y así podemos ver un baile tradicional de Bolivia realizado por

7 Henna es una palabra inglés, ampliamente utilizada en España para definir un tinte natural de color rojizo obtenido de la trituración de las hojas y del pecíolo del arbusto Lawsonia inermis. En muchos países de Asia, Oriente Medio y África del Norte, las mujeres lo utilizan para teñir el cabello y aplicar una técnica de coloración de la piel llamada en árabe mehandi (erróneamente definida "tatuajes de henna") empleada tanto con fines de belleza como para fines rituales.

8 Aunque anteriormente hemos hecho mención a la participación del colectivo gitano en la fiesta, seguimos afirmando que las actividades y los grupos participantes en la misma se organizan principalmente según sus lugares de procedencia y/o nacionalidades. En el caso de la comunidad gitana solo vimos que se tratase de un colectivo de gitanos españoles, sin relación - por ejemplo- con los cada vez más presentes en Granada, grupos de gitanos rumanos. Y en el caso de las asociaciones pro-inmigrantes, están integradas mayoritariamente por población no inmigrante nacional, salvo escasas excepciones. El tipo de actividades que estas entidades organizan es más bien para dar a conocer sus organizaciones, y las labores que realizan. En algunos casos sí observamos actividades que ponían en relación elementos pertenecientes a distintas comunidades, nacionales siempre. Sería el caso, por ejemplo, de una ONG que organizaba un taller de juegos de distintos países del mundo. 
personas de procedentes de Bolivia, o un té ofrecido por marroquíes. Aunque también hay excepciones, pues en una de las actuaciones en las que se bailaba una danza típica palestina las personas que bailaban, pertenecientes todos a una misma asociación, tenían procedencias nacionales diversas a pesar de que en las reuniones organizativas se hiciese referencia a ellos como a "los palestinos".

Pero el hecho de "nacionalizar" las actuaciones es una característica importante de la fiesta muy estimada por los participantes ya que, como nos decía una informante "la gente busca poder ver algo de su país, enriquecedor para las personas" (Participante del evento, Granada 2011). La Fiesta de las Culturas permite que se desplieguen toda una serie de tradiciones étnicas/nacionales que la gente muestra y comparte con los demás.

Desde nuestra posición como investigadoras es posible que quepa hacer críticas al respecto dado que, ni la diversidad tiene que ver sólo con la inmigración, ni las poblaciones inmigradas -y las autóctonas- tienen siempre identidades o culturas nacionales sin fisuras y muy bien definidas. Por esto estamos de acuerdo con Appiah cuando afirma: "I associate cultures with social groups not with nations because I want to insist again that a group of persons living together in a common state, under common authorities, need not have a common culture" (Appiah, 1994: 7). No obstante, en esta ocasión, queremos hacer otra reflexión. Y es que no podemos sino evidenciar que si este tipo de eventos son muy bien valorados por determinados segmentos de la población, es muy posible que sea porque esos mismos segmentos no se sientan representados e identificados en otros tantos eventos y efemérides de expresión folklórica y con más reconocimiento institucional, mucho más, que la Fiesta de las Culturas.

\section{Impacto: económico, político, social y comunitario}

Hemos realizado una descripción de lo que consideramos los elementos esenciales y/o más característicos del evento que estamos estudiando. Pero nuestro análisis no estaría completo si no tratásemos de analizar qué impacto tiene una política como esta en toda la sociedad granadina. A ello dedicamos el presente apartado.

No podemos negar que la Fiesta de las Culturas es un acontecimiento muy estimado y esperado en Granada por diversos colectivos de la ciudad. Es cierto que se trata de una iniciativa de mediana envergadura, donde los apoyos económicos e institucionales no son fuertes. Pero a pesar de las dificultades que ello pueda entrañar, sus organizadores están muy satisfechos con la continuidad en el tiempo que tiene, dado que año tras año -desde hace más de 15- se viene repitiendo. Pero nosotros nos hemos preguntado si esta "buena fama" con la que cuenta la fiesta se traduce en un impacto real sobre la población en distintos sentidos, y se traduce en un avance en entendimiento de las políticas interculturales como instrumentos dirigidos a toda la población con el objetivo de fomentar/facilitar la convivencia. Y las respuestas que hemos obtenido son de lo más variado. Veámoslo con más detenimiento.

El impacto de una política cultural siempre es difícil de evaluar, medir o estimar (Barbieri et al., 2011), en este caso además no contamos con indicadores cuantitativos para ello. Algunos de los organizadores lo manifestaban así:

[¿Qué impactos o resultados ha tenido el evento? ¿El evento contribuye a mejorar la imagen del barrio? ¿Y de los diferentes grupo?] Esto es muy difícil de decir, muy difícil [...] (Organizador del evento, Granada 2011b).

No obstante, el trabajo de campo nos ha permitido obtener cierta información al respecto. Y lo primero que queremos comentar es la difusión que ha tenido, que tiene habitualmente, el evento. Sabemos que las dimensiones del mismo son modestas, y que el apoyo 
institucional (autoridades locales) es también bastante cuestionable, dado que depende de coyunturas políticas concretas. Estos dos elementos han influido sin duda en que la publicidad y difusión de información sobre la celebración de la Fiesta de las Culturas no ayude en demasía a que la misma tenga un calado importante en toda la ciudad de Granada:

[¿Cómo se hace la difusión del evento? ¿A quienes está dirigido?] Hacemos una pequeña campaña con los carteles y sobre todo a través de las asociaciones que integramos, nos repartimos. Cada asociación tiene su propio ámbito de difusión, lo difundimos entre la gente nuestra [...] (Organizador del evento, Granada 2011b).

Vemos que todo el proceso de dar a conocer el evento depende de la "buena voluntad" de las entidades que participan en la organización y del trabajo que realizan personas a título individual. Las entidades organizadoras diseñan un cartel que distribuye por la ciudad (bares, sedes de las asociaciones, universidad, etc.) y a través del cual se publicita el evento. Esta forma de realizar las cosas no garantiza que la fiesta aparezca en las agendas culturales oficiales de la ciudad, y tampoco que tenga una cobertura en los medios de comunicación locales. De hecho, en la edición observada, no hubo presencia de los mismos. Podríamos decir que la publicidad que se hace se basa fundamentalmente en el "boca a boca", con todo lo bueno y/o lo malo que ello pueda tener. Pero antes de considerar que de esta forma se consigue llegar o no a mucha o poca gente, también hemos de preguntarnos ¿qué objetivos tienen al respecto los organizadores del evento? Y en este sentido vemos como en realidad existe una idea que subyace en muchas de las entidades organizadoras, y es que la fiesta ha de llegar sobre todo a la población inmigrada de nacionalidad extranjera:

Siempre hemos intentados hacer la fiesta en sitios, por lo menos teóricamente en sitios donde se facilitaras el acceso a inmigrantes, cuando lo hicimos en el Zaidín, uno de los argumentos básicos era que alli hay muchos inmigrantes, para posibilitarles el acceso, cuando nos fuimos al polígono... supuestamente por lo mismo [...] nos gusta hacerlo en la proximidad donde ellos están siempre para que posibilite que acudan más inmigrantes [...] (Organizador del evento, Granada 2011b).

Y en ese sentido podríamos considerar que el objetivo está bien trabajado. Sin embargo, no podemos dejar de insistir en que visibilizar a un colectivo menos empoderado es solo una parte de una gestión de la diversidad con pretensiones interculturales. De todas formas, también es cierto que no todas las personas encargadas de organizar el evento tienen dicha cuestión tan clara, ya que también vimos como quizá fijar el objetivo en que asistan "los inmigrantes" está más determinado por la dificultad que implica llegar a una pluralidad de personas:

Llegar a más gente, que se acerque gente distinta cada año es una de las dificultades con las que nos encontramos, que es también la razón porque movemos la fiesta por los barrios ¿no? (Organizador del evento, Granada 2011).

Que a un convencimiento claro sobre lo que tiene que perseguir una fiesta con pretensiones interculturales:

Yo creo que el objetivo de esta Fiesta de las Culturas es que llegue a todos, que no sea en especifico de inmigrantes, al contrario yo creo que debe llegar a 
más gente posible, que puedan participar diferentes organizaciones tanto las de aquí como de otros sitios (Organizadora de evento, Granada 2011).

Lo que sí nos ha quedado claro después de realizar el trabajo de campo es que la Fiesta de las Culturas no se plantea objetivos en el plano de lo económico, ni para las entidades organizadoras ni para los barrios:

[Nuestro interés] es mostrar a la comunidad como en muchas asociaciones aunque puedan tener trabajos individuales $e$ intereses individuales, pueden colaborar y pueden participar en un espacio para un bien común que no sea el interés propio, que sea el hecho de que muchas asociaciones se junten para un evento como este, en el que no ganen dinero, en que los grupos sea dedicar esfuerzos para ese objetivo, ¿no?... Que no cobren por ella, que no sea una actividad de la que se lucren sino todo lo contrario, ¿no? (Organizador del evento, Granada 2011).

La propia forma en que se organiza y se lleva a cabo todo el proceso (a partir de entidades sin ánimo de lucro, sin apenas apoyo institucional y económico por parte de las autoridades locales, sin actividades mercantilizadas...) es bastante reveladora al respecto: los objetivos -y los impactos, añadimos nosotras- son otros. Sin embargo, podríamos decir que sí tiene cierto impacto en el plano del desarrollo turístico de las zonas donde se realiza, aunque $a$ priori esto no sea uno de los objetivos con que se planifica el evento:

[...] no queremos que se convirtiera en algo turístico, una actuación para que [...] aunque con frecuencia lo que si sucede, si se acercan, se hace en una época en que hay bastante turistas en Granada, se acerca gente de fuera y suele haber una implicación muy interesante, gente de fuera de Granada, de otras provincias de España, de otros sitios de España, extranjeros inclusive, que han preguntado ¿esto qué es, esto de que va, por qué hacéis esto? y siempre ha sido un momento interesante explicarlo, hacerlo patente, pero no es que se hiciera con esta finalidad sino que se han acercado gente paseando (Organizador del evento, Granada 2011b).

Dado que el "folklorismo" con que se enfoca consigue atraer de manera fácil a viandantes y curiosos, testigos y participantes fortuitos que toman parte de ese, ahora sí, objetivo con que se plantea el evento: visibilizar poblaciones construidas como subalternas. Y precisamente la visibilización es el principal impacto que para nosotras, como investigadoras, tiene el evento, algo compartido por la mayoría de organizadores y participantes:

Si que contribuye a mejorar la visibilidad, la idea que la gente tiene de colectivos como de las personas migrantes, de las personas extranjeras. Bueno, que la gente entienda eso es muy relativo, y muy ambiguo. El cómo la gente entiende a la gente de otras culturas, depende de cada quien, pero sí que contribuye [a visibilizar], porque ven como un espacio de confluencia y un espacio de convivencia, gentes que atribuyen a otras culturas, y ven que están conviviendo y que están participando de los stands, de los talleres que se realizan, de los parlamentos públicos que se hacen, etc. Entonces sí que es positiva en ese sentido (Organizador del evento, Granada 2011).

Lo que ofrece la Fiesta de las Culturas es fundamentalmente un espacio donde interaccionar con los vecinos y vecinas -y demás personas asistentes- a través de actividades 
lúdicas, que de otra manera sería difícil de conseguir. Y tanto organizadores...

[...] es muy bonito ver que comunidades de vecinos o cualquier otra organización interesada en esto, llegue a convivir con toda la gente, con todas nosotras y nosotros, y es muy interesante [...] intentando trabajar sobre la integración (Organizadora del evento, Granada 2011b).

como asistentes...

[¿Piensa que estos eventos fomentan la convivencia entre los vecinos del barrio? ¿Entre los vecinos? Entre todos los que son de Granada, no solo del barrio este [...] [¿Y entre las personas de diferentes grupos culturales? ¿Por qué?] [Se ríe...] es evidente [...] (Participante del evento, Granada 2011).

... están de acuerdo con ello. Lo que se persigue es romper estereotipos, conseguir que la gente se conozca, crear precedentes para futuras relaciones, que la población establezca lazos de vecindad de cara a ir creando comunidad en espacios de creciente multiculturalidad, porque como nos decía - de forma muy esclarecedora- uno de los vecinos entrevistados "en el barrio está todo el mundo junto pero no revuelto" (Participante del evento, Granada 2011).

\section{Discusión y algunas notas para el debate}

Consideramos, por lo tanto, que los impactos más notables del evento estudiado sobre la comunidad tienen que ver con la visibilización de poblaciones no empoderadas. En cuanto a si esto se traduce o no en una convivencia real a largo plazo, así como en relaciones interculturales enriquecedoras para todas las personas implicadas, somos un poco más cautas. Por ello nos gustaría ahora aportar una serie de reflexiones acerca de por qué no interpretamos esto último como un logro pleno y/o inmediato del evento.

Lo primero que emerge en nuestros análisis realizados de la fiesta tiene que ver con la propia organización y planificación de la misma. Ya hemos apuntado anteriormente nuestra posición al respecto. La Plataforma pro-inmigrantes, como plataforma dinamizadora y organizadora del evento, está compuesta -como ya comentábamos- por entidades proinmigrantes y por asociaciones de inmigrantes. No obstante el papel y protagonismo que unas y otras tienen en decidir cómo será la fiesta es distinta, más bien desigual, en tanto que está vertebrada por relaciones de poder. Esta situación no es percibida de tal manera por las entidades pro-inmigrantes, pero sí por algunas de las asociaciones de inmigrantes, que consideran que las cosas podrían hacerse de otra forma:

[...] yo es que entendí la participación en la elaboración... en la difusión claro que contamos con toda la colaboración del entorno, con todos los recursos del entorno inmediato. Pero difundes algo que está planificado ya; para mi participación es cuando tú colaboras en lo que se genera, no en el resultado, participar en el resultado para mi es asistir; a no ser que en la misma fiesta se genere una actividad distinta a la que se han propuesto [...] si es participación, sino tú asistes y disfrutas de lo que te están ofreciendo entonces no, es planificado. Pero para mí la participación es participar desde la toma de decisiones [...] Entonces a eso me refería (Organizador del evento, Granada 2011).

Y quizá relacionado con lo anterior, una cuestión que también ya habíamos mencionado 
someramente, es que la fiesta ponga todo su esfuerzo en trabajar con un colectivo en concreto, la población -o deberíamos decir poblaciones-inmigrante(s), algo que no solo se hace de forma práctica, si no que se explicita en los discursos de los organizadores:

[...] mi responsabilidad en la fiesta es visibilizar que nuestra organización participa en ese Plataforma de inmigración, defender los objetivos de la fiesta defender la Plataforma pro-inmigrantes como una entidad abierta a la participación de todos esos colectivos, de los inmigrantes, fortalecer un poco la Plataforma ¿no? A través de la fiesta de las culturas (Organizador del evento, Granada 2011).

Entendemos que la interculturalidad hace referencia a la gestión de la diversidad, y las poblaciones inmigradas extranjeras solo representan una parte de la misma. Sin duda estamos hablando del colectivo más vulnerable en el momento actual, en tanto que carece de un estatuto jurídico que les proporcione derechos equivalentes a los del ciudadano o ciudadana nacional, sobre todo si estamos hablando de la inmigración procedente de países no comunitarios. Pero aún así solo representan una de las partes que componen la población granadina. Si entendemos que la diversidad es un recurso que debe gestionarse para contribuir al desarrollo de una ciudad (Zapata-Barrero, 2010), hemos de entender que no podemos parcelar la misma, sino que el camino es abordarla de manera conjunta. Lo contrario no está sino contribuyendo a diferenciar/desigualar aún más a aquellos que ya lo están, y frenando las posibilidades de una plena convivencia.

También hemos de mencionar, como cuestión sobre la que reflexionar, que la fuerza del evento se ponga en los elementos folklóricos. Es cierto que éstos son los que más público atraen, y que -en definitiva- se trata de un evento festivo. Pero también es cierto que -y este apunte no es solo para la Fiesta de las Culturas, sino para la gran mayoría de acciones que se identifican como culturales o interculturales- las políticas culturales habrían de expandirse, profundizar en más elementos y no quedarse en lo meramente folklórico. A continuación citamos las palabras de una chica que participó en la fiesta, justamente en uno de los bailes, y que comentaba:

Yo como observadora, y no solo de este año, sino también el año pasado, por eso me fijaba [...] me gustaría que fuese algo más que un baile solamente, que no se quede en baile, y pienso que mi responsabilidad como asociación es demostrar determinadas cosas de los países que participamos [...] para que se note que estamos integrados, de que por lo menos tenemos la intención de integrarnos, que formamos parte de lo que es [...] (Participante del evento, Granada 2011).

Ella reflexiona sobre la necesidad de mostrar el hecho de que su comunidad convive en el espacio en el que habita. Utiliza la palabra "integración", y se desprende de su discurso que siente la necesidad de que sus vecinos y vecinas, dejen de tener estereotipos sobre la falta de integración de los inmigrantes. Posiblemente eventos de este tipo puedan servir de plataformas en este sentido, espacios donde la gente tenga la oportunidad de conocerse y derribar ideas preconcebidas que impiden que se produzca o fluya la convivencia. Es decir, que la fiesta sirva para que las personas que habitan un mismo espacio se conozcan más.

Otra de las cuestiones sobre las que queremos llamar la atención es la asistencia y participación de los vecinos y vecinas en el evento. Y ya hemos hablado de las limitaciones que percibimos (y que nuestros informantes nos han señalado) en la forma de dar a conocer la celebración de la fiesta y que acaban siendo un condicionante para esa participación, dado que la publicidad no está todo lo respaldada que sería deseable por las autoridades locales: 
[...] se debería de mejorar, la gente no sabe... iyo no sabía que era hoy! nos hemos enterado por el pasacalles, que era en pro de los derechos de los inmigrantes, nos hemos acercado y nos hemos encontrado con esto [...] (Participante del evento, Granada 2011).

En la edición observada no podemos decir que la participación vecinal fuese escasa (más bien fue modesta), pero sí que nos gustaría señalar que en otras ocasiones se han tenido grandes problemas con ello. A continuación citamos un comentario que nos hacía una de las personas entrevistadas con respecto a la fiesta celebrada el año anterior al observado:

[...] el año pasado lo hicimos en el polígono de Cartuja, en el parque 28 de febrero, también pensábamos que era un sitio ideal y bueno la primera parte, la parte de los talleres fue muy bien pero la segunda parte de los conciertos fue muy mal, muy mal porque realmente inmigrantes se quedaron muy pocos, poquísimos y el objetivo fundamental de la convivencia entre inmigrantes y locales pues no se consiguió en la fiesta, porque no hubo inmigrantes, problemas añadidos, pero en fin [...] (Organizador del evento, Granada 2011b).

De nuevo nos hace poner la atención sobre el énfasis que se hace en que participen las poblaciones inmigrantes. Creemos que es muy interesante la cita anterior, porque nos muestra muy claramente cómo se percibe problemático para la convivencia el que esté ausente una de las partes que conforman la diversidad de los barrios. El entrevistado nos decía que el evento resultó "mal porque realmente inmigrantes se quedaron muy pocos"; y continúa diciendo: "el objetivo fundamental de la convivencia entre inmigrantes y locales pues no se consiguió". Y decimos que es curioso, interesante, porque no se ve de igual manera cuando los que faltan son "los de aquí", los vecinos nacionales autóctonos ¿por qué sucede esto? Muy probablemente porque existe una tendencia a considerar la Fiesta de las Culturas como "la fiesta de los inmigrantes" y cuando éstos faltan ¿qué hacemos?

Y no podemos dejar de realizar una evaluación crítica del evento en relación al apoyo institucional que recibe el mismo. Ya hemos mencionado algunas cuestiones al respecto. La Fiesta de las Culturas responde claramente a un formato de evento cuyo mayor impulso corresponde al tercer sector. Son entidades sin ánimo de lucro, conformadas por la propia sociedad civil quienes promueven, planifican y ejecutan el evento y además sin ningún objetivo económico y/o mercantil. Es cierto que la participación es desigual entre dichas entidades, ya que unas tienen un papel más fuerte en el plano de las decisiones de planificación y otras son invitadas por las primeras a participar. Creemos que es algo positivo que sean este tipo de organizaciones quienes se dediquen a estos menesteres, aunque sin duda se echa en falta un apoyo más explícito de las autoridades locales:

[Esto es] una iniciativa que es popular y participativa... Yo considero que, evidentemente, cuanto más apoyo, no solo financiero, sino institucional, pues mejor. Lo que pasa es que eso condiciona a veces, pero sí que debiera haber más apoyo, este año apenas hay ¿no? Y eso repercute en las posibilidades de traer gente de organizar cosas y en la difusión también ¿no? [...] Este año creo que no nos subvenciona ni el Ayuntamiento, ni la Diputación. Sí que es cierto que en esta época de crisis, entrecomillado, donde las instituciones no tienen liquidez pues [...] (Organizador del evento, Granada 2011).

Una práctica que dependa de la voluntariedad de aquellos que la ponen en marcha no puede convertirse, por muy buenas intenciones que encontremos en ello, en una política cultural con calado en toda la sociedad. Y a los hechos nos remitimos: depende de quien 
gobierne que se disponga de más o menos recursos, que se disponga de un mejor o "menos adecuado" espacio para celebrar el evento, que exista la posibilidad de una mejor o peor difusión del mismo, incluso, y creemos que este es el momento de dejar constancia de ello, se pueden llegar a bloquear las acciones que dan lugar al propio evento. Veamos el siguiente relato, donde uno de los organizadores entrevistados nos cuenta cómo en ocasiones, y como consecuencia de una falta de apoyo muy evidente por parte de las autoridades locales, se ha intentado que deje de celebrarse la fiesta:

[...] nosotros siempre hemos hecho la fiesta en sitios céntricos de Granada, al principio la hacíamos en la Plaza de las Pasiegas, un sitio ya tradicional, reunía todas la condiciones que nos interesaban, hasta que un año simplemente nos dijeron que no. Creemos que fue también por denuncias de unos vecinos que hay por allí fascistas, claramente fascistas contra los inmigrantes y nos lo prohibieron absolutamente. A partir de entonces andamos todos los años con el problema de encontrar un lugar donde hacerla, esta año todavía -tú lo sabes- no sabemos donde será [al día de la entrevista, faltan 10 días para la realización del evento...], nos han denegado la explanada del Palacio de Congresos y estamos todavía esperando que nos den alternativas... si es que nos dan algunas [...] pero bueno, esto nos ha pasado varias veces, siempre hemos conseguido un sitio al final, con el apoyo -y tenemos que decirlo claramentesiempre de IU que siempre han apoyado clarísimamente, porque el PSOE nunca se ha mojado, nunca ha apostado nada por este tema, a lo largo de los años hemos hecho muchas denuncias, de actuaciones de la policía local, tanto cuando estaba el PSOE que con el PP, hemos hecho muchas denuncias y esto ha hecho que no seamos gratos a las autoridades (Organizador del evento, Granada 2011b).

No es posible avanzar con estas limitaciones de base. Aunque el problema de fondo pese a lo que pueda parecer- creemos que no es fundamentalmente de corte partidista; es decir, que no tiene que ver exclusivamente con colores políticos. El problema es más estructural, nace de las relaciones desigualitarias de poder que vertebran nuestras instituciones. Estamos totalmente de acuerdo con la visión del problema que nos daba una de las personas entrevistadas, cuando nos decía que:

[...] se quiere apoyar otros tipos de actos, es la realidad, así de claro vamos: no lo apoyan porqué quieren apoyar otro tipo de cultura [...] cultura que sea Lorca, flamenco, religión, y ya está. Y la feria [...] y, hombre, hay otras cosas que también son culturales ¿no? [...] que el ayuntamiento apoye todos los eventos culturales religiosos, católicos, y no apoye este evento cultural pues eso es un reflejo, del tipo de cultura que se quiere mostrar [...] (Organizador del evento, Granada 2011).

Nos resultan unas palabras muy esclarecedoras, que nosotras matizamos afirmando que existe un claro interés por apoyar oficialmente algunas expresiones culturales -las que tienen que ver con el folklore, básicamente- cuando estas emanan de grupos concretos que habitan la ciudad -los situados en posiciones hegemónicas-.

Pero ¿y qué sucede con la convivencia? Nuestra conclusión es que la fiesta trabaja de manera positiva en este aspecto, salvando los apuntes que hemos hecho anteriormente. Es cierto que finalmente el evento es percibido por mucha de la gente que participa en el mismo como "la fiesta de los inmigrantes". Sin embargo, ello no significa que, aunque con un alcance limitado, las personas que se acercan a pasar un rato en la fiesta -inmigrantes o au- 
tóctonos- estén generando lazos de vecindad con quienes posiblemente no lo hacen de forma habitual. Un ejemplo de ello nos lo muestra una participante de nacionalidad boliviana:

[Este tipo de actividades] claro que podrían mejorar la convivencia, porque a veces pasamos desapercibidos ¿por qué? Pues porque no se nos conoce. Siempre hablando desde mi experiencia personal, pero de una boliviana se sabe que trabaja en una casa y no se sabe si después tiene vida social; porque también tenemos vida social, y me gustaría tener más, pero es una lástima que no se dé la oportunidad [...] yo creo que todas personas extranjeras tenemos el derecho de disfrutar de nuestra cultura (Participante del evento, Granada 2011).

Y otro un chico español:

[¿ंPiensa que estos eventos fomentan la convivencia entre los vecinos del barrio?] Claro entre los vecinos y entre todos los que son de Granada, no solo del barrio este (Participante el evento, Granada 2011).

Y durante nuestra observación del evento pudimos ver muchas muestras de ello: chicas marroquíes enseñando a bailar la danza del vientre a chicas españolas, señoras mayores de distintas procedencias participando juntas en un taller, familias enteras participando en los bailes organizados por las mujeres bolivianas, un grupo de chicos y chicas de lo más diverso que prepararon una batucada.

\section{Notas conclusivas}

El reto de las políticas culturales en una sociedad multicultural es conseguir visibilizar y dar protagonismo a todas las opciones culturales, en un contexto donde tradicionalmente la homogeneidad ha sido el objetivo (Rodríguez, 2012). Si estamos en el plano de lo lúdicofestivo, sería más positivo que se generaran eventos donde autóctonos e inmigrados hicieran exposición pública de bailes, gastronomía y demás elementos folklóricos de manera conjunta, compartiendo, reflejando con ello todas las diversidades y de-construyendo la existencia de folklores hegemónicos y folklores subalternos. Si diferenciamos entre "nuestras fiestas" y las "fiestas de los inmigrantes" el resultado nunca podrá ser la convivencia plena. Es necesario encontrar elementos comunes que identifiquen y aglutinen a la población en un contexto de diversidad:

"Se trata de gestionar la pluralidad de interacciones que se producen en un contexto de diversidad con el objetivo social de la cohesión, pero también con el objetivo político de contribuir a la creación de un contexto común compartido y de una cultura pública común" (Zapata-Barrero, 2008:101).

Pero, por otro lado, es también urgente tener en cuenta más aspectos/dimensiones de la diversidad y ampliar el concepto de interculturalidad incorporándolo a la experiencia de la vida cotidiana, y entendiéndolo como un sentimiento de convivencia, no como algo que se experimenta solo en eventos y/o actividades puntuales.

Para que se pueda generar una convivencia que garantice el respeto mutuo es necesario "romper con las relaciones de subordinación entre culturas" (Cruz, 2014: 251). Y esto no puede depender de la "voluntad subjetiva" de quien gobierna en un momento determinado, sino que ha de estar presente en las estructuras: políticas, económicas y sociales; que son, 
en definitiva, las que condicionan las relaciones entre personas en el día a día. Por esto la justicia cultural no puede ser separada de la justicia social (Viaña, Claros y Sarzuri, 2009).

Finalmente queremos remarcar la necesidad de seguir reconsiderando los conceptos de cultura y diversidad cultural, dado que defendemos que un equivocado entendimiento de los mismos tiene consecuencias sociales negativas (García et al. 2012; Olmos y Contini 2015, en prensa). Ya hemos apuntado a lo largo del presente artículo a una excesiva folklorización del evento estudiado. Incluso, hemos hecho referencia a que son muchos quienes demandan este tipo de actividades: festivas, lúdicas, folklóricas... y que parecen atraer a mucho público.

Si bien entendemos la necesidad de considerar y fomentar estos tipos de actividades, consideramos que una forma de entender la gestión de la diversidad cultural como exhibición de elementos tenidos por característicos pero presentados como si fueran algo congelado en el tiempo, fotografías fijas, puede encubrir algún riesgo. La propia dinámica de funcionamiento de las culturas difiere de estos presupuestos dado que ésta se encuentra en constante movimiento, transformación, contagio, modificación... Y quizás sería precisamente esto lo que habría que celebrar en este tipo de eventos para no correr el peligro de reforzar estereotipos, marcar diferencias y generar relaciones de alteridad entre personas que ahora comparten un mismo espacio y que tan solo con esto ya están construyendo cultura. Sospechamos que de cómo se haga la gestión de esas relaciones pueda depender el resultado final, la emergencia de nuevas representaciones y procesos culturales de tipo convivencial o en forma de nuevas exclusiones.

\section{Bibliografía}

Appiah, K. A. (1994). Identity against culture: Understandings of Multiculturalism. Berkeley, CA: University of California Berkeley.

Barbieri, N., Partal, A., y Merino, E. (2011). Nuevas políticas, nuevas miradas y metodologías de evaluación: ¿cómo evaluar el retorno social de las políticas culturales? Papers: Revista de Sociología, 96(2), 477-500.

Castells S. y Miller, M. J. (2003). The age of Migration. New York: Guilford Press.

Clark, T. N. y Navarro, C. (2009). Governing Cultural Issues and Scenes: Towards the Emergence of a 'Local Cultural Policy Domain'? En City Future '09 Conference EURA/UAA, Madrid, 4-6 de Junio de 2009. Recuperado de http://www.cityfutures2009.com/PDF/39_Navarro_Clemente.pdf.

Contini, P. (2015). La diversidad en la ciudad. Un análisis comparado de la convivencialidad en dos barrios de Granada. En prensa, 1-16.

Contini, P. y Olmos, A. 2015. La gestión de la diversidad cultural después del Estado-nación: ¿estamos ante el declive del contenido nacional de Estado? En Santos, B. De S. y Cunha, T. (Eds.), Actas Coloquio Internacional Epistemologias do Sul: aprendizagens globais Sul-Sul, Sul-Norte e Norte-Sul, Vol.2 (pp. 281-298). Coimbra: Centro de Estudos Sociais. Universidade de Coimbra.

Cruz Rodríguez, E. (2014). Multiculturalismo, interculturalismo y autonomía. Estudios Sociales, 22 (43), 241-269.

García Castaño, F. J., Olmos Alcaraz, A., Contini, P. y Rubio Gómez, M. (2011). Criticas y superaciones conceptuales en la gestión de la diversidad cultural. En Gualda Caballero, E. (Ed.), Inmigración, ciudadanía y gestión de la diversidad (pp. 31-65). Sevilla: Universidad Internacional de Andalucía.

García Castaño, F.J., Olmos Alcaraz, A. y Contini, P. (2012). La gestión de la diversidad cultural en Andalucía: cuando hablar de interculturalidad es hablar de inmigración. En Monereo Pérez, J.L., García Castaño, F. J., y Fernandez Avilés, J. A., La inmigración en Andalucía. Instituciones, aspectos jurídicos-sociales y culturales (pp. 599-618). Granada: Comares.

Lofland, L. H. (1998). The Public Realm: Exploring the City's Quintessential Social Territory. New York: Aldine de Gruyter. 
Meissner, F. y Vertovec, S. (2015). Comparing superdiversity. Ethnic and Racial Studies, 38(4), 541-555.

Olmos Alcaraz, A. y Contini, P. (2015). Las ausencias del paradigma intercultural en España: un análisis a propósito de experiencias de diversidad en contextos urbanos multiculturales. En prensa, 1-26.

Oliveira, N. y Padilla, B. (2012). A diversidade como elemento de desenvolvimiento/atracao nas políticas locais urbanas: contrastes e semelhancas nos eventos de celebracao intercultural. Sociologia, Revista da Faculdade de Letras da Universidade do Porto, 22, 129-162.

Padilla, B., Azevedo, J. y Olmos Alcaraz, A. (2015). Superdiversity and conviviality: exploring

frameworks for doing ethnography in Southern European intercultural cities. Ethnic and Racial Studies, 38(4), 621-635.

Ray, B. (2003). The role of cities in Immigrant Integration. En Migration Information Source. Recuperado de http://www.migrationpolicy.org/article/role-cities-immigrant-integration.

Real Instituto Elcano de Estudios Internacionales y Estratégicos (2004). La política cultural en Espa$\tilde{n} a$. Madrid: Real Instituto El Cano de Estudios Internacionales y Estratégicos.

Rodríguez Morató, A. (2012). El análisis de la política cultural en perspectiva sociológica. Claves introductorias al estudio del caso español. Revista de Investigaciones Políticas y Sociológicas, 11, 15-38.

Santos, B. de S. (2011). Introducción: las epistemologías del Sur. En CIDOB (Ed.), Formas-Otras. Saber, nombrar, narrar, hacer. IV Training Seminar de Jóvenes investigadores en Dinámicas interculturales (pp.9-22). Barcelona: CIDOB.

Vertovec, S. (2007). Super-diversity and its implications. Ethnic and Racial Studies, 30(6), 10241054.

Viaña, J., Claros, L. y Sarzuri, M. (2009). Presentación. En Mora, D. (Dir.), Interculturalidad crítica y descolonización. Fundamentos para el debate (pp. 7-9). La Paz: Convenio Andrés Bello.

Zallo, R. (2011). Análisis comparativo y tendencias de las políticas culturales de España, Cataluña y País Vasco. Madrid: Fundación Alternativas.

Zapata-Barrero, R. (2008). Diversidad y Política pública. Papeles de relaciones ecosociales y cambio global, 104, 93-104.

Zapata-Barrero, R. (2010). Diversidad y política cultural. La ciudad como escenario de innovación y de oportunidades. Barcelona: Icaria. 